\title{
Corrosion Assessment of Zn-Rich Epoxy Primers With Carbon Nanotube Additions in an Electrolyte With a Bacteria Consortium
}

\author{
Homero Castaneda ${ }^{*}$ and Monica Galicia ${ }^{2}$ \\ 1 Department of Materials Science and Engineering, Texas A\&M University, College Station, TX, United States, \\ ${ }^{2}$ Departamento de Ciencias Químico Biológicas, Instituto de Ciencias Biomédicas, Universidad Autónoma de Ciudad Juárez, \\ Ciudad Juárez, Mexico
}

Multifunctional coatings for corrosive environments have been developed to include two basic principle protection mechanisms: a barrier mechanism acting as a mass transfer process blocker, and cathodic protection mechanism acting as a charge transfer process promoter. Both mechanisms were assessed for Zinc-Rich Epoxy (ZRE) coatings in the presence of Carbon Nanotubes (CNTs) and exposure to a bioelectrolyte in order to study the evolution process during microbial corrosion conditions. The purpose of this study is to characterize in a comprehensive experimental platform the electrochemical

OPEN ACCESS

Edited by:

Michele Fedel,

University of Trento, Italy

Reviewed by:

Farhad Sharif,

Amirkabir University of

Technology, Iran

Idalina Vieira Aoki,

University of São Paulo, Brazil

${ }^{*}$ Correspondence:

Homero Castaneda

hcastaneda@tamu.edu

Specialty section:

This article was submitted to

Environmental Materials,

a section of the journal

Frontiers in Materials

Received: 09 July 2019 Accepted: 14 November 2019 Published: 03 December 2019

Citation:

Castaneda H and Galicia M (2019)

Corrosion Assessment of Zn-Rich

Epoxy Primers With Carbon Nanotube

Additions in an Electrolyte With a

Bacteria Consortium.

Front. Mater. 6:307.

doi: 10.3389/fmats.2019.00307 response of a dual-protection zinc epoxy coating with different ratios of additive carbon nanotubes to active zinc particles upon exposure to a sulfate reducing consortium. Carbon nanotubes addition was found to affect both the prevailing mechanism at the coating interfaces and the formation of a biofilm at the coating surface that influenced the relatively dominance of the barrier protection mechanism. These multifunctional coatings with active particles could help to balance the charge transfer efficiency in terms of the sacrificial of zinc and barrier mechanisms, which influence biofilm formation and have potential consequences for biocorrosion on carbon steel.

Keywords: zinc rich epoxy coatings, carbon nanotubes, biocorrosion, biofilm, EIS

\section{INTRODUCTION}

Most coatings used for microbiologically induced corrosion (MIC) in industry are designed to provide an effective barrier to inhibit corrosion processes and/or provide a biocidal effect. These coatings are synthetized using organic, inorganic, and hybrid approaches. Organic coatings have been demonstrated to provide an effective physical barrier in a variety of aggressive conditions (Deflorian et al., 2005; Abdolahi et al., 2014; Fedel et al., 2019). However, when exposed to anoxic conditions such as those prevailing in seawater or oil and gas pipelines, some of these coatings degrade faster upon exposure to either a bacterial consortium or a specific strain of microbial attack (Enning and Garrelfs, 2014).

Unfortunately, inorganic and hybrid protective coatings on steel have also been proven to degrade when exposed to MIC in such anoxic conditions (Abdolahi et al., 2014; Enning and Garrelfs, 2014; Ciubotariu et al., 2015). Environmentally friendly coatings are being developed to improve their physicochemical properties to resist microbiological attack, either in aerobic or anaerobic conditions, while avoiding toxicity to the environment from the inherently toxic formulations of some coatings. 
Recent coating research has investigated nano-architected sacrificial coatings as a new technology with dual protection mechanisms. The first is galvanic or cathodic protection, which is achieved by integrating electrochemically active particles into the organic coating matrix. The second protection mechanism is the classical barrier effect offered by a polymeric matrix. The addition of nanostructures such as carbon nanotubes (CNTs) can enhance both properties by influencing the interconnectivity of active particles and filling the voids created during synthesis of the coating in the polymeric matrix, as noted by Cubides and Castaneda (2016).

Zn-rich epoxy primers have shown excellent results and have been used by the industry since the 60's and 70's (Weinell and Rasmussen, 2007). The mechanisms of corrosion protection in the Zn-rich epoxy primers include a galvanic effect due to metallic zinc dissolution, as well as a barrier effect resulting not only from the epoxy resin but also via the formation of $\mathrm{Zn}$ and Fe products (Deflorian et al., 2005; Weinell and Rasmussen, 2007). However, a balance must be considered in the formulation in order to assure cathodic protection for an extended time. A load of $90 \mathrm{wt} . \%$ of Zn particles may be beneficial for the charge transfer process (Park and Shon, 2015). However, that amount can reduce substrate adhesion and cause problems with the spraying application related to the high viscosity and poor dispersion of zinc particles (Park and Shon, 2015). Hence, research has sought to decrease the percentage of $\mathrm{Zn}$ load while retaining the connection among the particles and maintaining or increasing mechanical coating properties (Weinell and Rasmussen, 2007; Jeon et al., 2013; Park and Shon, 2015).

Weinell and Rasmussen (Weinell and Rasmussen, 2007) noted that different pigments, such as thixotropic agents, can be added to the epoxy matrix of coatings to prevent zinc precipitation. The authors incorporated colored pigments to differentiate between steel substrate and the grayish $\mathrm{Zn}$ primer, adding oilabsorbing pigments to decrease the $\mathrm{Zn}$ load while maintaining the same level of cathodic protection. Park and Shon suggested the addition of multi-walled carbon nanotubes (MWCNTs) to a maximum load of 0.6 wt.\%, which resulted in improved conductivity, adhesion strength, and corrosion protectiveness as the MWCNTs increased from 0 to 0.25 wt.\% (Park and Shon, 2015). Similarly, the addition of MWCNTs in an epoxy coating provided greater adhesion strength, transport behavior, and hydrophobicity as the MWCNT concentration increased from 0 to 0.5 wt.\% (Jeon et al., 2013).

The microbiological environment and underlying mechanisms have not been extensively explored in coating studies of Zn-rich epoxy primers. A few studies have evaluated Zn-rich epoxy primers in media containing sulfate reducing bacteria (Liu et al., 2007; Wang et al., 2012), but to the best of our knowledge no studies have investigated $\mathrm{Zn}$-rich primers with MWCNTs added to their matrix in an environment including a sulfate reducing consortium.

This work aims to determine the electrochemical behavior of a Zn-rich primer with MWCNTs additions in an electrolyte containing an SRM consortium. Electrochemical techniques were used to elucidate the interfacial mechanisms. We used electrochemical impedance spectroscopy as a primer technique
TABLE 1 | Specifications of the different coating systems.

\begin{tabular}{lcc}
\hline Coating & Zinc (wt.\%) & (CNTs) $(\sim$ wt. \%) \\
\hline ZnR & 70 & 0 \\
Zn-1CNT & 70 & 1.0 \\
Zn-2CNT & 70 & 2.0 \\
\hline
\end{tabular}

to characterize the mechanisms presented at different interfaces formed in the system, and the results were analyzed using electrical analog element coupled with high-resolution surface techniques. Different CNTs compositions were used to support the influence of the mechanism's dominance based on the corrosion control method.

\section{MATERIALS AND METHODS Microbial Culture}

The consortium of sulfate reducing bacteria (sulfate reducing microorganisms) used in this study was collected with an inline inspection tool used for internal inspection in pipelines (the so-called smart 'PIGS"). The sample consortium was inoculated in ATCC1250 modified Baar's medium for Sulfate Reducers with $\mathrm{NaCl}\left(2.5\right.$ wt.\%) [ATTC ${ }^{\circledR}$ ]. The $\mathrm{pH}$ value was adjusted to 7.2 under de-aeration by applying nitrogen to the electrolyte. The medium was autoclaved at $120^{\circ} \mathrm{C}$ for $15 \mathrm{~min}$. The SRM consortium was incubated at $37^{\circ} \mathrm{C}$.

\section{Multifunctional Coatings}

The Zn-rich epoxy primers and multi-walled carbon nanotubes were supplied by Tesla NanoCoatings company. The epoxy resin (Tesla P1150ASAS) was mixed with CNTs at 1 and 2 wt.\%. After the CNTs were completely dispersed in the epoxy resin through mechanical stirring according to a previously method (Cubides and Castaneda, 2016), Zn particles at 70 wt.\% were slowly added while stirring the solution, and a hardener (Tesla P1150BSAS) was then poured in to a final weight ratio of 2:1 (epoxy resin-hardener). The 70 wt. \% was used based on the previous work (Cubides and Castaneda, 2016) where is was demonstrated the $\mathrm{Zn}$ content was the most balanced for cathodic protection or sacrificial conditions. In addition, Zn-rich epoxy primer without CNTs was also used in this study. The coatings were air-sprayed onto UNS1008 steel plates. This procedure was performed previously (Cubides and Castaneda, 2016). Table 1 shows the different sample characteristics.

\section{Electrochemical Characterization}

A three-electrode electrochemical cell was used for the experimental characterization. The electrochemical cells were autoclaved. The working electrode was the multifunctional coating sample. The counter electrode was a platinum mesh, and the reference electrode was a saturated calomel electrode (SCE). The working electrodes and the reference and auxiliary electrode were sterilized with $70 \%$ ethanol and acetone and set under UV light and laminar flow. They were placed on a $25 \mathrm{~mL}$ electrochemical cell containing culture medium under $\mathrm{N}_{2}$ gas injection before each experiment. It was conducted 


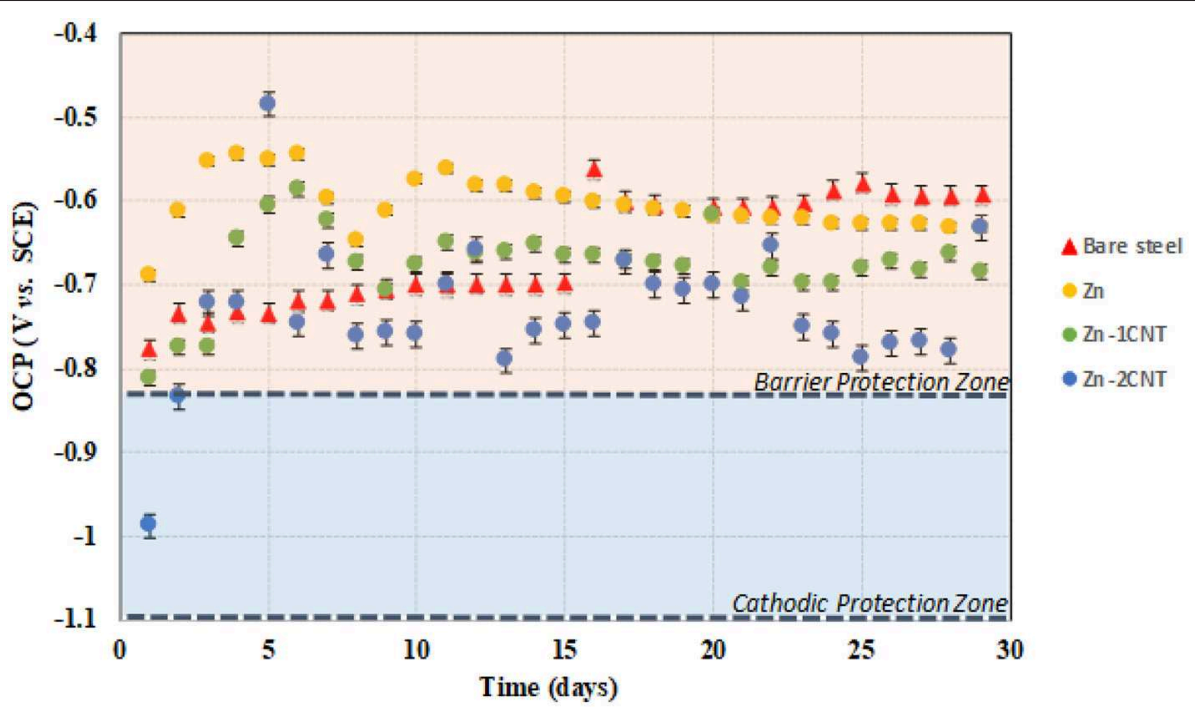

FIGURE 1 | OCP for bare steel and the three different multifunctional coatings under bioelectrolyte conditions.

at $25^{\circ} \mathrm{C}$. OCP was performed on prior EIS measurements during a period of $10 \mathrm{~min}$. EIS was performed in a frequency range of $100 \mathrm{KHz}$ to $10 \mathrm{mHz}$ with a $\pm 10 \mathrm{mV}$ peak-to-peak amplitude $v s$. the open circuit potential (OCP). All the tests were carried out in a potentionstat/galvanostat (VSP-300; Bio-Logic Science Instruments), using the software EC-Lab ${ }^{\circledR}$ V.10.32. All electrochemical experiments were performed in duplicate to ensure reproducibility of the electrochemical behavior.

\section{SEM Sample Preparation}

Once the electrochemical test was completed, the samples were rinsed with $\mathrm{PBS} 1 \mathrm{X}(8.0 \mathrm{~g}$ of $\mathrm{NaCl}, 0.2 \mathrm{~g}$ of $\mathrm{KCl}, 1.4 \mathrm{~g}$ of $\mathrm{Na}_{2} \mathrm{HPO}_{4} \cdot 2 \mathrm{H}_{2} \mathrm{O}$, and $0.2 \mathrm{~g}$ of $\mathrm{KH}_{2} \mathrm{PO}_{4}$ per liter, $\mathrm{pH}=7.2$ ). Subsequently, the samples were fixed with glutaraldehyde $2.5 \%$ $\mathrm{w} / \mathrm{v}$ in PBS for $2 \mathrm{~h}$, with washing every $30 \mathrm{~min}$. All fixation was conducted at room temperature. After fixation, the cells were washed twice in PBS and then re-suspended in sterilized ultrapure water to avoid salt crystallization during the drying process. The samples were dried for $24 \mathrm{~h}$ in a LABCONO Freeszone 6 lyophilizer. Finally, the samples were sputtered with silver particles and observed under a JSM-7000F/JOEL microscope through energy-dispersive spectroscopy (EDS).

\section{RESULTS AND DISCUSSION}

\section{Open Circuit Potential}

The ZnR coating system shows the potential evolution influenced by different conditions and interfacial configurations such as the epoxy physical barrier, the galvanic phenomenon between zinc and steel, the physicochemical barrier of corrosion products, the concentration of $\mathrm{Fe}$ and $\mathrm{Zn}$ near the particle/coating or substrate/coating interfaces and inside the coating, amongst other factors, as described by Cubides (Cubides and Castaneda, 2016). The cathodic protection of steel has been established at $\leq-0.90 \mathrm{~V}$ vs. $\mathrm{Cu} / \mathrm{CuSO}_{4}(-0.826$ vs. SCE) under anaerobic conditions (Schwerdtfeger, 1958; Guan et al., 2016; Liu and Cheng, 2017). This level is drawn as a dash line in Figure 1. At higher (more positive) potentials, barrier protection tends to be the dominant control mechanism, which may be associated with the epoxy-polymer and the corrosion products of $\mathrm{Zn}$ and/or Fe. The OCP monitoring of the AISI/SAE 1008 steel substrate and the multifunctional coatings under biotic conditions is shown in Figure 1. The OCP for the bare steel is more positive (passive region or protective layer region based on thermodynamics) than the cathodic potential criteria because the galvanic effect results in a negative potential value, in which the steel faces immune thermodynamic conditions. The OCP for the bare steel 1008 was determined in a test where the sample was kept in the culture medium in bacterial consortium conditions, and the steel potential was found to be influenced by the biofilm and corrosion products formed at the surface of the substrate. Classic work by Castaneda (Castaneda and Benetton, 2008) characterized mild steel in seawater in the presence of SRB, which resulted in positive potentials due to the combination of corrosion products and biofilm. Figure 1 shows the initiation and growth stages for the biofilm formation. At this respect, Castaneda marked 14 days for the initiation to biofilm growth transition, similar to this condition, where the OCP increases the magnitude following 2 weeks of exposure.

The Zn-rich primer (70 wt.\% Zn) exposed to the bacteria consortium shows a potential oscillating within the barrier protection zone over the exposure time. This means that the Zn particles are randomly distributed in the epoxy matrix with a connectivity between them that is insufficient to trigger the galvanic effect formation. There is a transient state during the first 10 days of exposure, and during this time, the potential values are in the barrier zone. This behavior is associated with water uptake in the coating and the formation of $\mathrm{Zn}$ products during the initial days of exposure. Finally, the potential is around the OCP of 1008 carbon steel in biotic conditions, but both experimental 
results are above the corrosion protection located in the barrier protection zone, and barrier zone dominance can result from the $\mathrm{Zn}$-Fe corrosion products and/or biofilm formation to produce a blocking effect for the water uptake process.

At the early stage, the $\mathrm{Zn}-1 \mathrm{CNT}$ potential is located close to the cathodic protection zone for the first day. Following the negative potentials, a transient stage occurs during the next 10 days within the barrier protection area. The initial conditions could be the response of the bioelectrolyte wetting over the $\mathrm{Zn}$ particles and due to the interconnectivity among $\mathrm{Zn}$ particles and steel arising from the influence of MWCNTs or the cathodic protection effect; the potential between 4 and 10 days may be the result of $\mathrm{Zn}$ and/or Fe corrosion products acting as a barrier or passive particle state, which switches the system to prevail in more positive potentials. These latter compounds fill the porous surfaces within the coating structure, and the potential subsequently increases at day 2 . The interaction between consortium/coating subsequently changes the quantity and morphology of the products, and the potential decreases at day 10 . Eventually, the potentials reach $\sim-0.680 \mathrm{~V}$ vs. SCE in the barrier zone, $100 \mathrm{mV}$ more negative than the $\mathrm{ZnR}$ sample. Subsequently, the potential remains stable at close to $-0.680 \mathrm{~V}$ vs. SCE. This latter effect could be due to the influence of the biofilm at the surface, in which the zinc ions inhibit the formation of the biofilm layer at initial conditions (Babich and Stotzky, 1978; Bong et al., 2010; Billanger et al., 2015; Tong et al., 2015), but the ions are depleted and the biofilm starts to grow over time to become more homogeneous and distributed compared with the 2CNT, and the magnitude of the potential becomes more negative than the $\mathrm{ZnR}$ sample to produce a less dominant barrier effect with the addition of 1CNT.

OCP for the $\mathrm{Zn}-2 \mathrm{CNT}$ sample are more negative than the threshold for the cathodic protection magnitude during the first 2 days. This behavior is associated with a greater MWCNTs concentration, resulting in better interconnection between the $\mathrm{Zn} / \mathrm{Zn}$ electrical connection particles due to the CNT/Zn/CNT interconnectivity. This latter finding is confirmed in the crosssection SEM images. These potentials are more negative than the Zn-1CNT results. A transient potential state exists between days 3 and 10 of exposure. After 10 days, there are two transient states during the barrier protection zone and close to the cathodic protection threshold, one between 10 and 15 days and the other between 16 and 24 days. Finally, the potential keeps fluctuating in the barrier protection zone and reaches lower values than the OCP of carbon steel in biotic conditions. The potentials reveal the dominance of cathodic protection for this sample in different stages. Particle activation was promoted by the distribution of CNTs within the coating, and the products formed in the coating are not sufficiently compact to stop bioelectrolyte uptake within the coating, with the unstable biofilm leading to lower potential magnitudes. This may result from greater electrochemical activity among particle/coating interface. This latter result is evidence of a certain grade of galvanic effect, i.e., the system is at a lower potential than the value found for steel without coating due to reaction with the $\mathrm{Zn}$ particles. The presence of two increments may be due to the creation of products that fill the pores in the coating, with the decrease resulting from the particle layer and an interaction forming with an incipient heterogeneous biofilm. That is, zinc ion formation inhibits biofilm growth and leads to various areas of biofilm depletion, thus affecting the morphology of these compounds over time and altering the distribution of the biofilm at the surface of the coating.

\section{Qualitative Analysis of Impedance}

Figure 2 shows the complex and phase angle diagrams for Bare steel and Zinc Rich Epoxy multifunctional coatings in SRM consortium environment.

The Nyquist representation for the AISI 1008 bare steel sample exposed to the biotic electrolyte is shown in Figure 2A. The results show a loop with a large diameter characteristic of a capacitance and charge transfer process. The phase angle representation is illustrated in Figure 2B. The medium frequency response is associated with capacitance (double layer) at the surface, whereas the response at low frequency is associated with the electrochemical reactions at the steel/electrolyte interface. On day 5, this system represents biofilm formation, and the corrosion products form an inorganic and organic mixing layer. At low frequencies, the magnitude of the semicircle's diameter is associated with the substrate/mixing layer/electrolyte interface. The magnitude for the biotic conditions results in a loop intercepting the locus point on the order of $12,000 \Omega$ $\mathrm{cm}^{2}$, whereas at 10 days, an increase in the loop diameter occurs, which may be associated with the accumulation of a mixing layer formed at the surface. At the surface, the number of active sites decreases, and the Rct increases, as previously described by Castaneda and Benetton (2008), with mild steel exposed to an SRM electrolyte during the first 2 weeks. At day 15 , the impedance magnitudes begin to decrease, reflecting an activation mechanism, and an increase in the number of active sites occurs at the metallic substrate/mixing layer interface. On day 20, the impedance lowers the magnitude for this sample, an effect associated with the active sites, owing to the charge transfer process. After 25 days, the contribution of two potential interfaces is revealed by two maximum points in the phase angle representation. At days 25 , and 29 , the active sites remain constant, owing to the maintenance of the impedance at low magnitudes and the formation of two separate contributions at the interface layers, owing to the stability and physical presence of a mixing layer on the surface, as a result of precipitation of corrosion products and presumed biofilm formation. This finding corroborates the OCP biofilm transition from initiation to growth stage. The EC representation for this system can be described by Figures 3A,B; the first days include an activation capacitance RC analog, and after 25 days, two time constants are represented by two constant phase elements.

\section{Zinc Rich Epoxy}

The EIS results for the ZnR sample are shown in Figures 2C,D. Two loops are observed: one at medium-high frequency and another at low frequency in the complex representation. In the phase angle representation, two time constants are observed, representing the interfaces due to the presence of a mixed layer (biofilm, epoxy coating) and the zinc particle/coating interface. Figure 3B represents the equivalent circuit used for the $\mathrm{ZnR}$ 

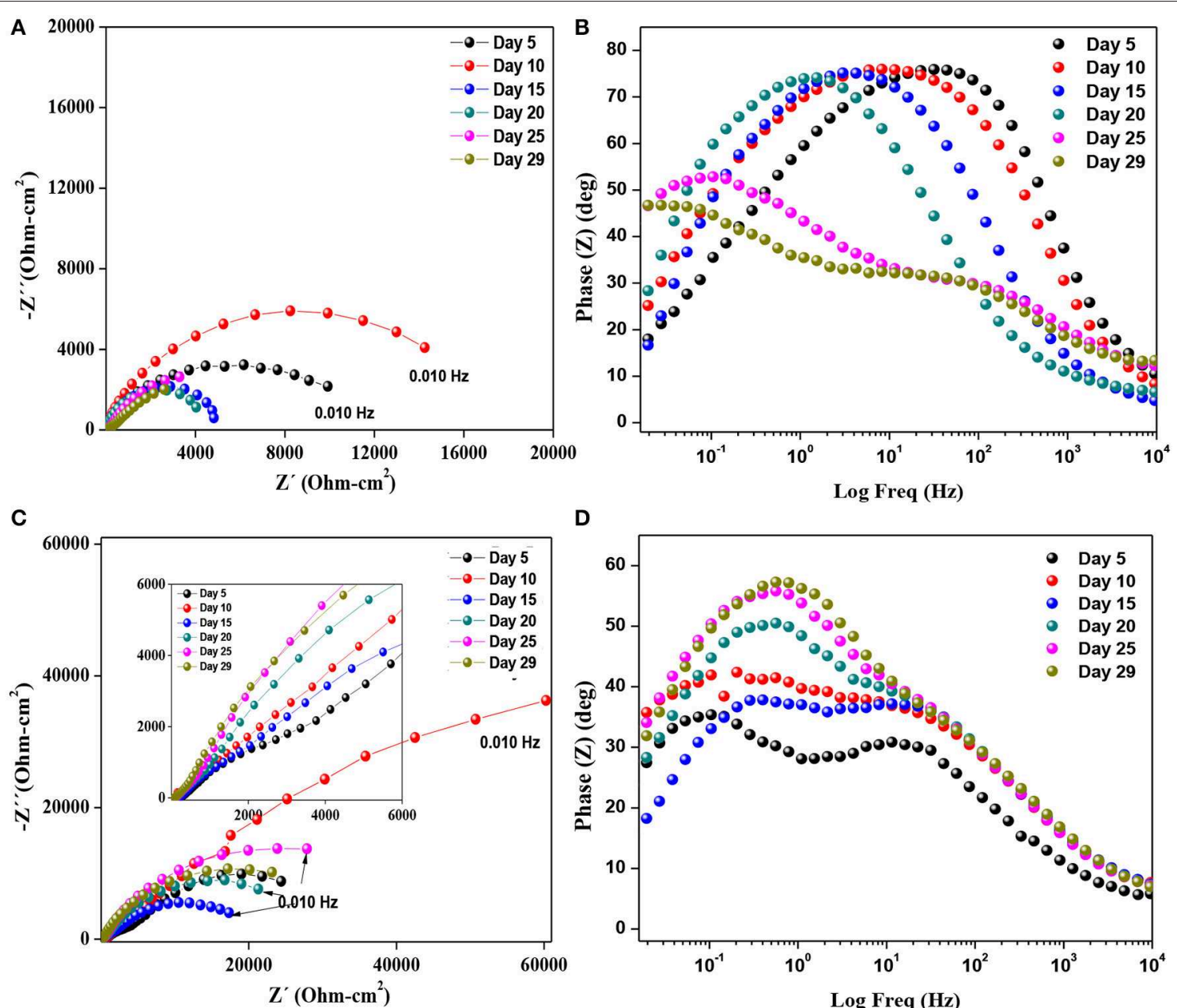

D

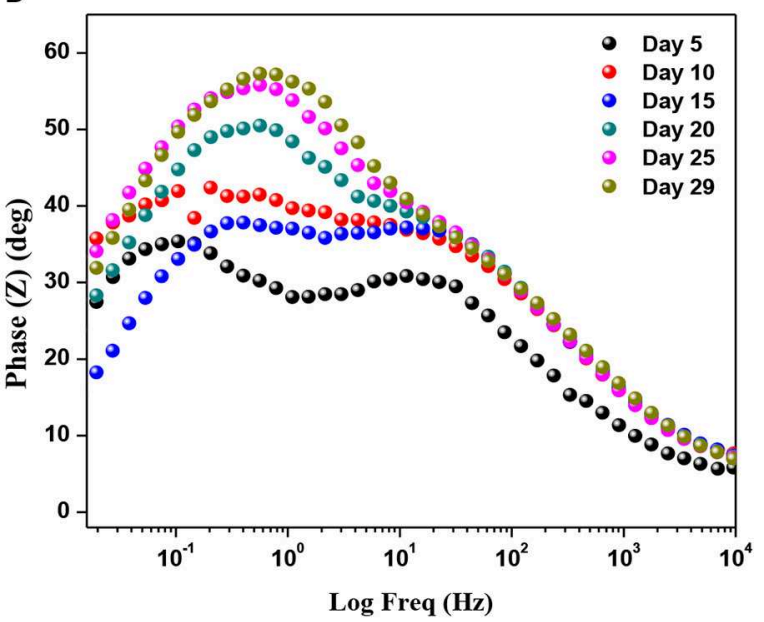

E

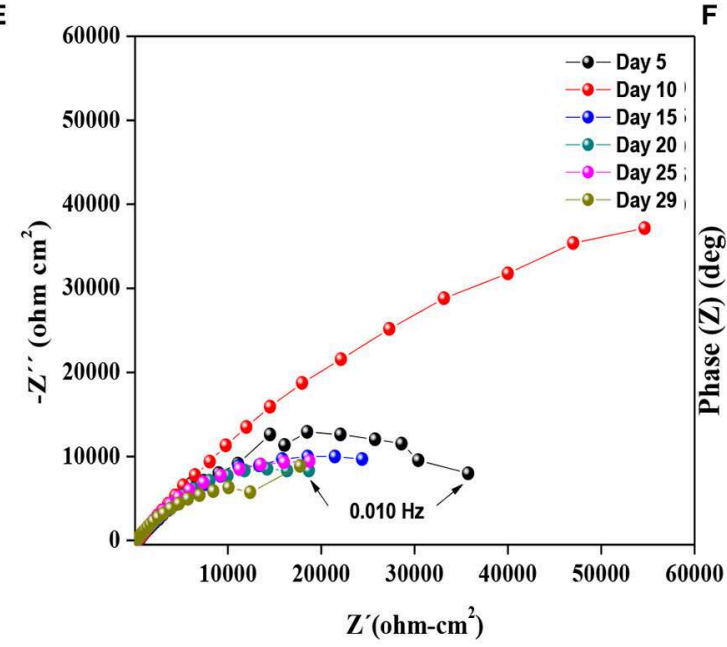

$\mathbf{F}$

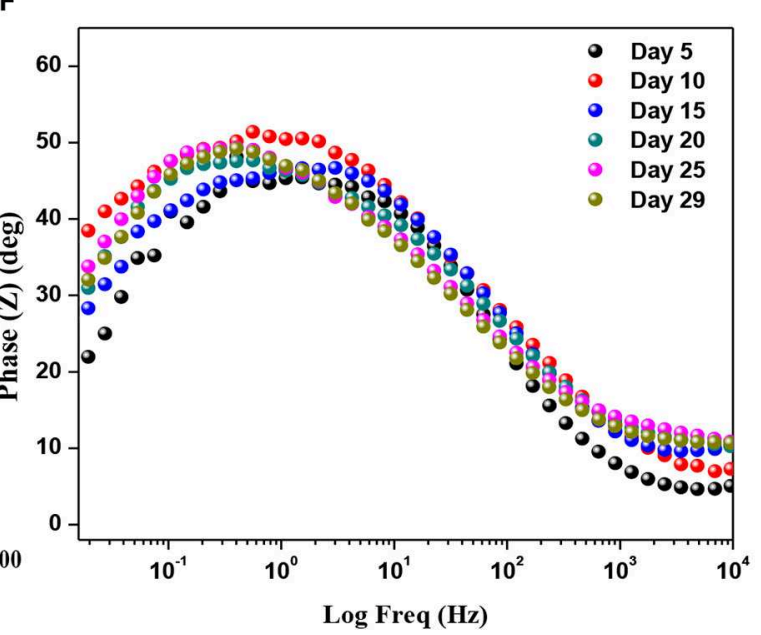

FIGURE 2 | Diagrams of Nyquist and phase angle in the SRM consortium for (A,B) 1008 bare steel, (C,D) for ZnR coating, and (E,F) for Zn-1CNT coating.

epoxy/steel interface in the early stage. Part of this circuit has been proposed by Cubides to represent the $\mathrm{ZnR}$ on a metallic substrate system when the zinc particles are active after being exposed to an electrolyte, where $R_{\mathrm{s}}$ is the electrolyte resistance, $R_{\mathrm{p}}$ is the porous layer resistance formed by the coating, $\mathrm{CPE}_{1}$ is the constant element for medium frequencies, $R_{\mathrm{ct}}$ is the 
charge transfer resistance due to the active particle/coating interface, $\mathrm{CPE}_{2}$ is the constant phase element associated with the double layer capacitance, and $\mathrm{n}$ is the roughness factor (Cubides and Castaneda, 2016). In general, all the impedances are higher than those of the bare steel samples, as can be seen in Table 2. This is due to conductivity of the Zn-rich epoxy influenced by the polymer coating matrix. In addition, the impedance signal is influenced by several factors such as electrolyte uptake within the coating, the galvanic effect of the $\mathrm{Zn}$ particles, the relation between $\mathrm{Fe} / \mathrm{Zn}$ areas exposed to electrolyte, product formation, and biofilm formation (Abreu et al., 1999; Galicia et al., 2017). The $R_{\mathrm{ct}}$ impedances in this case are within an order of magnitude at around $50,000-100,000 \Omega-\mathrm{cm}^{2}$ as illustrated in Table 2. This is in concordance with the OCP results in which the behavior of the systems is maintained at a specific potential zone. The semicircle acquired at the highermedium frequency is associated with presumable coating-biofilm mixed layer, and the semicircle recorded at low frequency is the response of $\mathrm{Zn} /$ Epoxy interfacial reactions. The equivalent circuits describing the early stage of the system are illustrated in Figure 3B. The EC shows two time constant phase elements in parallel with two resistances representing early and continued evolution for 20 days. The time constants associated with the medium and low frequencies are consistent with the evolution of each element in the system. During the initial days, the electrolyte uptakes the coating and wetting of $\mathrm{Zn}$ particles, even though the galvanic effect prevailing at the particle/coating interface the overall OCP shows positive magnitudes away from the cathodic protection potential for anaerobic conditions, this latter can be attributed to the poor particles interconnection within the epoxy, resulting in a small area for the electrochemical reaction. The zinc particles react to produce ionic species and corrosion products; the zinc ionic species preserve the surface of the coating with no biofilm formation, likely due to a cytotoxic
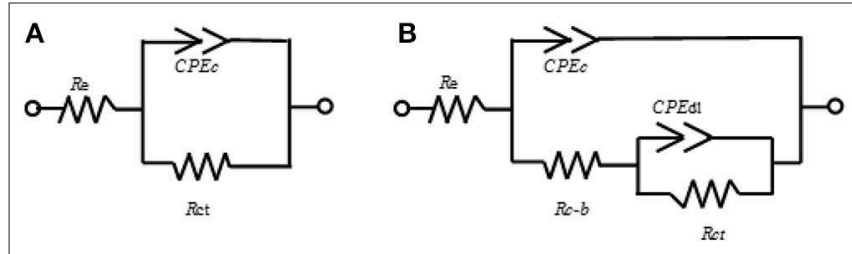

FIGURE 3 | Equivalent circuits proposed for the interface evolution on the basis of the interfacial components. mechanism against microorganisms (Babich and Stotzky, 1978; Billanger et al., 2015; Tong et al., 2015). Two time constants are maintained for the phase angle representation while the $R_{\mathrm{p}}$ and $R_{\mathrm{ct}}$ impedance in the complex representation increases up to 20 days. The wetting of the zinc particles following the charge transfer reactions no longer predominates, owing to the formation of corrosion products such as $\mathrm{ZnO}$ and $\mathrm{Zn}(\mathrm{OH})_{2}$ at the zinc particle/coating interface, as reported previously (Cubides and Castaneda, 2016). The production of extracellular polymeric substances (EPSs) apparently begins to influence the high impedance values revealed at high-medium frequencies. Subsequently, a mature biofilm forms a heterogeneous layer as illustrated in Figure 4a obtained by scanning electronic microscopy, SEM. After 20 days, the complex signature reaches a higher impedance magnitude, resulting in the capacitance EIS signature representation. This latter result is consistent with the phase angle representation with the formation of only one time constant at medium-low frequencies. The final layer is a mixture of EPS/biofilm, corrosion products, and precipitates, which allows the capture of the influence of this layer in the frequency interval range. The phase angle identifies one time constant in the system with a magnitude of 60 degrees, closer to the ideal capacitor effect of 90 degrees. This latter is attributed to a semi compact (heterogeneous) layer formed by mixture of organic epoxy, organic EPS/biofilm and corrosion products. The sample was evaluated at 29 days of bioelectrolyte exposure conditions. The Zn-rich epoxy illustrated in Figure 4a shows that the morphology is modified after exposure and demonstrates the underlying mechanisms; the sample shows the presence of a heterogeneous Extracellular Polymeric substances on the electrode as a biofilm. The balance between the $\mathrm{Zn}$ presence and the biofilm formation produce a biolayer of $12.5 \mu \mathrm{m}$ in thickness.

\section{Zinc Rich Epoxy With 1CNT}

The Nyquist diagram of the $\mathrm{Zn}-1 \mathrm{CNT}$ is displayed in Figure 2E for the complex representation and Figure $2 \mathrm{~F}$ for the phase angle. There is one loop with a finite locus point in the real axis, owing to the capacitance behavior and charge transfer reaction step control process at the zinc/coating interface. At day 5, the $R_{\mathrm{ct}}$ impedance magnitudes in the EIS signature are similar in magnitude to those of the ZnR epoxy samples (see Tables $\mathbf{2}$ and $\mathbf{3}$, respectively). The electrical interconnection between $\mathrm{Zn}$ particles is increased with the addition of CNTs, which in turn favors the formation of products within the coating through favoring the charge transfer reaction at the $\mathrm{Zn} /$ coating interface, and the level

TABLE 2 | Fitting parameters from an equivalent circuit simulation for AISI 1008 bare steel.

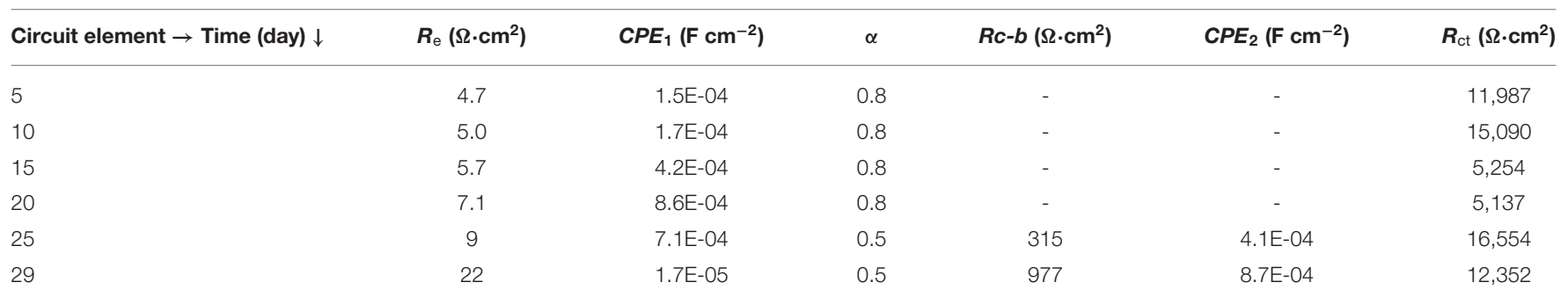



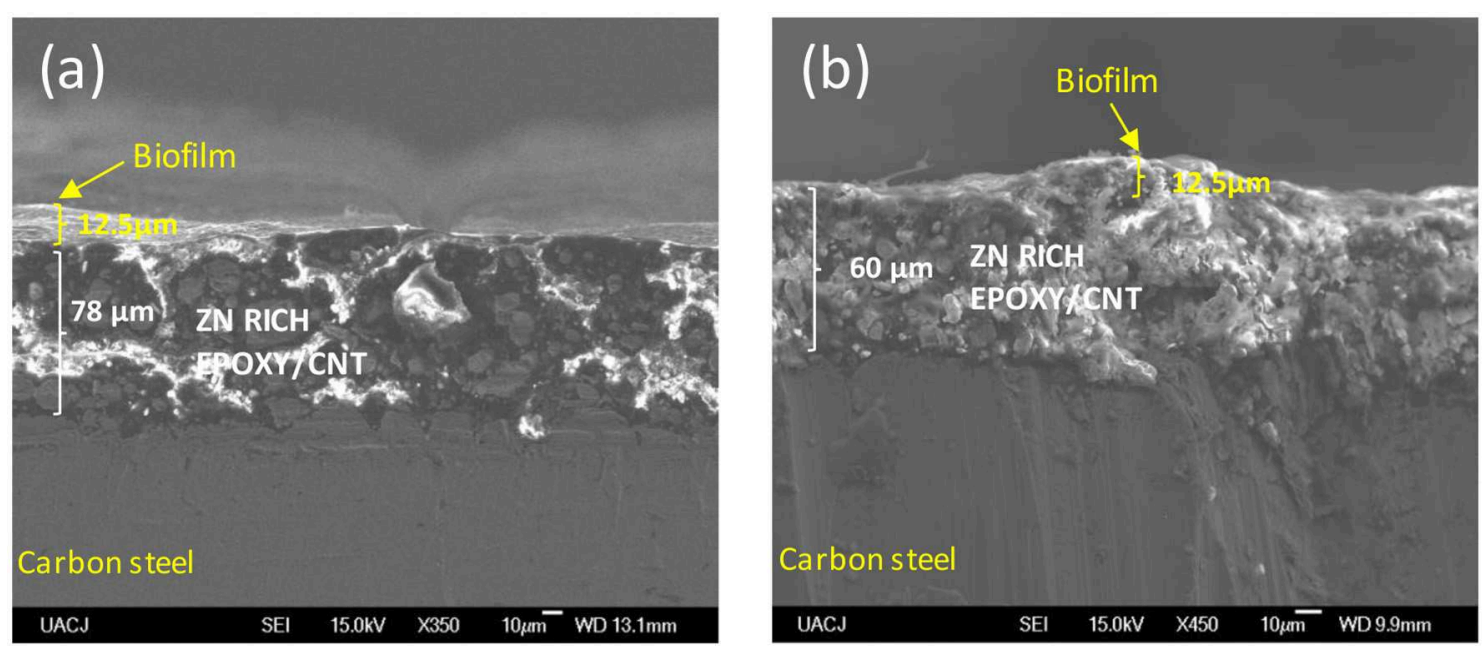

FIGURE 4 | Cross-section SEM images of the coatings immersed in an electrolyte with the SRM consortium. (a) Zn rich-primer, (b) Zn-rich primer with 1 wt.\% CNTs.

TABLE 3 | Fitting parameters from an equivalent circuit simulation for multifunctional coatings.

\begin{tabular}{|c|c|c|c|c|c|c|}
\hline Circuit element $\rightarrow$ Time (day) $\downarrow$ & $R_{\mathrm{e}}\left(\Omega \cdot \mathrm{cm}^{2}\right)$ & $\mathrm{CPE}_{1}\left(\mathrm{~F} \mathrm{~cm}^{-2}\right)$ & $\alpha$ & $R c-b\left(\Omega \cdot \mathrm{cm}^{2}\right)$ & $C P E_{2}\left(\mathrm{~F} \mathrm{~cm}^{-2}\right)$ & $\boldsymbol{R}_{\mathrm{ct}}\left(\Omega \cdot \mathrm{cm}^{2}\right)$ \\
\hline \multicolumn{7}{|l|}{$\mathrm{ZnR}$} \\
\hline 5 & 502 & 8.5E-07 & 0.5 & 21204 & 5.7E-07 & 39,143 \\
\hline 15 & 205 & $9.8 \mathrm{E}-07$ & 0.5 & 23631 & 1.3E-06 & 1,203 \\
\hline 20 & 171 & $1.2 \mathrm{E}-04$ & 0.6 & 29509 & $5.8 \mathrm{E}-07$ & 5,654 \\
\hline \multicolumn{7}{|l|}{$\mathrm{Zn}-1 \mathrm{CNT}$} \\
\hline 5 & 400 & 8.9E-07 & 0.6 & - & - & 39,001 \\
\hline 10 & 426 & $5.4 \mathrm{E}-07$ & 0.6 & - & - & 50,038 \\
\hline 15 & 183 & 1.0E-02 & 0.6 & - & - & 37,817 \\
\hline 20 & 139 & $1.4 \mathrm{E}-02$ & 0.6 & - & - & 33,855 \\
\hline 5 & 290 & 5.0E-07 & 0.6 & - & - & 77,908 \\
\hline 10 & 157 & 1.0E-05 & 0.6 & - & - & 35,247 \\
\hline 15 & 125 & 6.7E-05 & 0.6 & - & - & 43,741 \\
\hline 20 & 123 & 7.2E-05 & 0.6 & - & - & 42,736 \\
\hline 25 & 138 & 9.2E-05 & 0.6 & - & - & 43,580 \\
\hline 29 & 49 & 3.1E-04 & 0.6 & - & - & 13,532 \\
\hline
\end{tabular}

of protection subsequently improves, as corroborated by the OCP in early stages. The presence of the CNTs promoted zinc particle area/substrate or particle/particle interactions; initially, charge transfer process kinetics are favored due to the area available for galvanic reactions. The initial presence and distribution of extracellular polymeric substances was influence by $\mathrm{Zn}$ leaching and precipitation within the coating as well as precipitation at the surface of the corrosion products. EPS formation and subsequent biofilm stability was influenced by the amount of reacted zinc at the particle/coating interface. Following system evolution, changes in the corrosion products and growth of the biofilm shifted the charge transfer magnitude. Competition between formation of the biofilm on the surface and the corrosion products within the coating is balanced by the formation of zinc ions and influenced by the amount of CNTs. The SEM image in Figure $\mathbf{4 b}$ shows a smaller biofilm thickness on the coating surface after 29 days of exposure when the concentration of CNTs stayed at $1 \mathrm{CNT}$. The impedance gain could mark a transition moment of the system, which does not follow the EIS application principle of stability (Bosch et al., 2001) because the 
OCP for the sample decreased significantly at 10 days. However, the subsequent exposure times at 15,20, and 25 days exhibit an increase in charge transfer resistance (see Table 3), or $R_{\mathrm{ct}}$, which agrees with the OCP results. The 1CNT concentration influences the EPS, biofilm formation and mix layer, by dissolution or inhibition mediated by the $\mathrm{Zn}$ ions and the kinetics of the corrosion products formed at the $\mathrm{Zn}$ particle interface. This latter result is due to the decrease in $\mathrm{Zn}$ ionic formation caused by surface de-activation of particles and their depletion resulting from their usage in the formation of corrosion products, with the decrease also due to biofilm inhibition (Babich and Stotzky, 1978; Bong et al., 2010). The presence of one time constant in the phase angle representation for $\mathrm{Zn}-1 \mathrm{CNT}$ reflects the influence of capacitive behavior resulting from a mixture of the organic coating, inorganic corrosion products and biofilm. This indicates that a mix layer was formed on top of the coating, as evidenced by the impedance signature of Figure $2 \mathrm{~F}$ and SEM image of Figure $\mathbf{4 b}$. The morphology of the biofilm is very different from that of with zinc-rich sample. The $\mathrm{Zn}$ products were obtained with a higher density, the corrosion products expanded the ZnR coating in some locations, and the biofilm was no continuous compared with $\mathrm{ZnR}$. For $\mathrm{Zn}-1 \mathrm{CNT}$, the phase angle shows one-time constant appearance at early stage. The early stage at OCP correlates interconnectivity of the CNTs, with active zinc particles reacting faster compared to without the CNTs.

\section{Zinc Rich Epoxy With 2 CNT}

The impedance signature of the $\mathrm{Zn}-2 \mathrm{CNT}$ sample is exhibited in Figures 5A,B. There is one loop for the complex representation and one time constant for the phase angle signature. The electrical connection among the $\mathrm{Zn}$ particles is evident in the OCP results, and this is associated with a $\mathrm{Zn}$ area that is much greater than the Fe iron exposed to the electrolyte, which is due to the increased CNTs content. The EIS signature corroborates the formation/initiation of EPS and the biofilm as well as the charge transfer dominance due to $\mathrm{Zn}$ particle activation, resulting in corrosion products within the coating. The formation of corrosion products at the particle/coating interface should be favored due to the higher electronic interconnectivity. However, the biofilm does not form homogeneously due to the presence of $\mathrm{Zn}$ products formed from bulk zinc ion generation from the coating and their transport to the coating surface. The substantial CNT content surpasses the ionic $\mathrm{Zn}$ content that inhibits biofilm formation. After 10 days, the impedance magnitude decreases, owing to the activation of Zn particles. At day 29, the system evolves to the lowest charge transfer resistance, $R_{\mathrm{ct}}$ magnitude. The CNT increases the Zn particle kinetics and avoids continuous formation of a mixed layer that does not appear at this concentration.

The impedance analysis gave insight into the competition among anodic dissolution of zinc particles, EPS/biofilm formation, and the formation of zinc-rich corrosion products. Phase angle representation shows one time constant. The signature is characteristic of capacitive process and controlled by charge transfer resistance. The time constant at medium frequencies may represent the influence of a capacitance of a unified (mixed) layer forming at the surface, and the low frequency characterizes the particle/coating interface. With time, anodic dissolution of $\mathrm{Zn}$ and the formation of $\mathrm{Zn}$ corrosion products become less prevalent, and thus do not provide sufficient protection to the metal substrate. From the Nyquist plot, the impedance of the $\mathrm{ZnR}$ coating increased with time. Great influence in resistance is attributed to the EPS/biofilm formation noted above in combination with inorganic semiconductor $\mathrm{ZnO}$ and $\mathrm{Zn}(\mathrm{OH})_{2}$ corrosion products following $\mathrm{Zn}$ dissolution, which provides added barrier. EIS analysis agrees with the OCP measurement findings in that the addition of CNTs to the polymeric matrix structure improves the electrical connectivity between zinc particles and the metallic steel substrate through electron transport. In early stages, both the $\mathrm{Zn}-1 \mathrm{CNT}$ and $\mathrm{Zn}-2 \mathrm{CNT}$ coated samples showed potential below the CP threshold potential. SEM images after 28 days of immersion in Figure 7, suggest that the biofilm forms at the early days of immersion and subsequently grows/inhibits based on the $\mathrm{Zn}$ content generated by the more efficient galvanic system. The limited presence of an exopolysaccharide matrix, along with the limited population of bacteria and other organisms, accounts for the possibility that biofilm limited formation at the surface occurred, in part due to the presence of carbon nanotubes for $\mathrm{Zn}-1 \mathrm{CNT}$ and $\mathrm{Zn}-2 \mathrm{CNT}$.

The SEM image of a Zn-2CNT sample in Figure 6 shows several regions with biolayer removal and some residual $\mathrm{Zn}$ particles in the epoxy. This Zn-2CNT sample has the thinnest randomly distributed biofilm formed at the top of the coating surface. This latter result, could be attributed to the dissolution of zinc particles, which form $\mathrm{ZnCl}_{2}$ or $\mathrm{ZnO}$ that presumably inhibit initial biofilm formation by poisoning certain cellular elements present in microorganisms (Tong et al., 2015). Many investigations have demonstrated that zinc cations, zinc oxides and zinc chlorides are cytotoxic by disturbing gene regulation processes associated with the production of extracellular polymeric substances (EPSs) (Babich and Stotzky, 1978; Billanger et al., 2015; Tong et al., 2015). The above factors prevent construction of the matrix component of the biofilm. OCP and EIS results were able to demonstrate initiation of the biofilm or mixed layer formation due to the transient state revealed by both techniques over time. The shifts in OCP, magnitude and impedance elements associated with the system interfaces accompanied trends including formation of the biofilm is a clear indicator of the influence by the CNT in terms of corrosion control mechanism. Zn ions formed following CNT addition, which are then transported into the coating and inhibit biofilm formation in locations with a higher density of $\mathrm{Zn}$ particles, the $\mathrm{Zn}$ and CNT content can be balance to establish a critical magnitude or Zn/CNT ratio for the efficiency of the ZRE epoxy coatings.

\section{Surface Evolution (Top and Cross Section) Characterization Before and After Bioelectrolyte Exposure}

Figure 7 shows the cross-section SEM images obtained for the multifunctional coatings after 29 days of immersion in the 

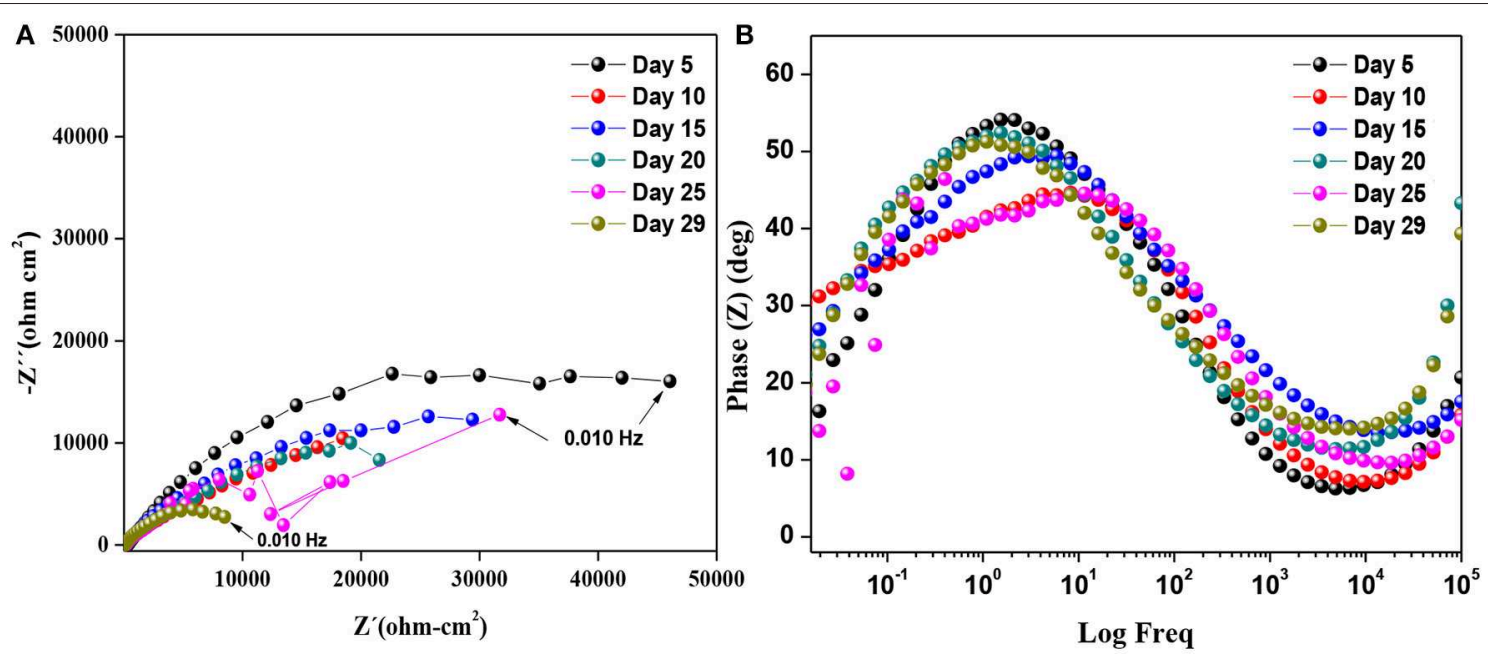

FIGURE 5 | Diagram of Zn-2CNT coating in the SRM consortium: Nyquist (A) and phase angle (B) representations.
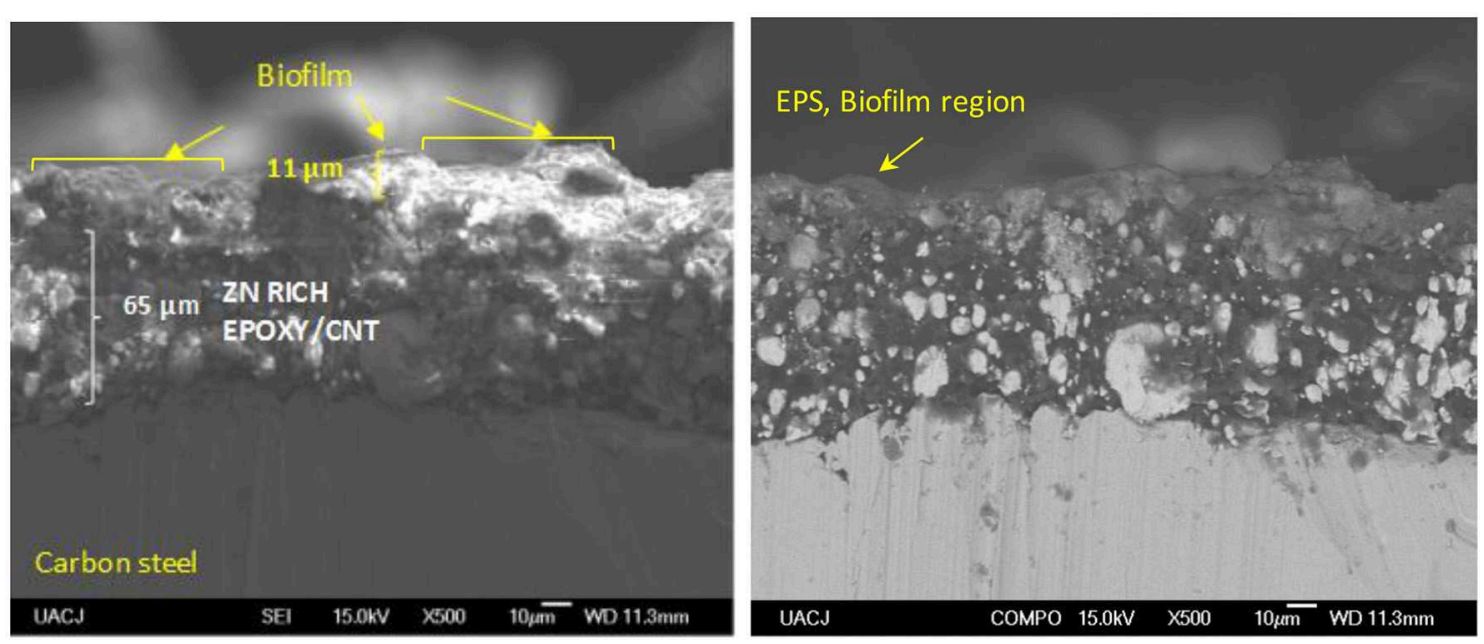

FIGURE 6 | Cross-sectional SEM images of the coatings immersed in an electrolyte with the SRM consortium. Zn-rich primer with 2 wt.\% CNTs. Morphology of the image and a back scattered electron image.

SRM bioelectrolyte. These images corroborate the findings from the OCP data and the impedance analysis. For Zn-1CNT and $\mathrm{Zn}$-2CNT coatings, it was possible to detect and demonstrate the presence and interconnectivity of $\mathrm{Zn}$ particles with carbon nanotubes at the interface of carbon steel, the coating matrix itself and the EPS/corrosion product layer. The biofilm layer can be considered to provide greater extension for the $\mathrm{ZnR}$ coating because, as described above, the CNT content leads to cytotoxicity toward the main bacterial and fungal constituents of the SRM consortium (Upadhyayula and Gadhamshetty, 2010). The increase in CNT content promotes the formation of $\mathrm{Zn}$ ions, thus resulting in a cytotoxic environment. The images in Figure 7 reveal biofilm patch formation instead of a continuous layer at the electrode surface. Therefore, the presence of biofilm is not favored when CNTs are present.
The presence of CNTs, as evident in Figure 7, was associated with an excellent influence on conductivity at the inner section of the coating according to the impedance analysis. Lack of uniform layer is ascribable to the dissolution of zinc cations, which are presumably cytotoxic to the microorganisms. In turn, zinc ions could be also responsible for the formation of corrosion products such as $\mathrm{ZnO}$ and $\mathrm{ZnCl}_{2}$, also the presence of these corrosion products also became evident according to the EDS elemental analysis, this latter illustrated in Figure 8.

Figures 8a-c show top-view SEM images of the three multifunctional coatings after 29 days of immersion in bioelectrolyte. For $\mathrm{ZnR}$ in Figure 8a, a continuous layer of extracellular polymeric substances is observed covering the surface, which becomes less evident in the presence of CNT 


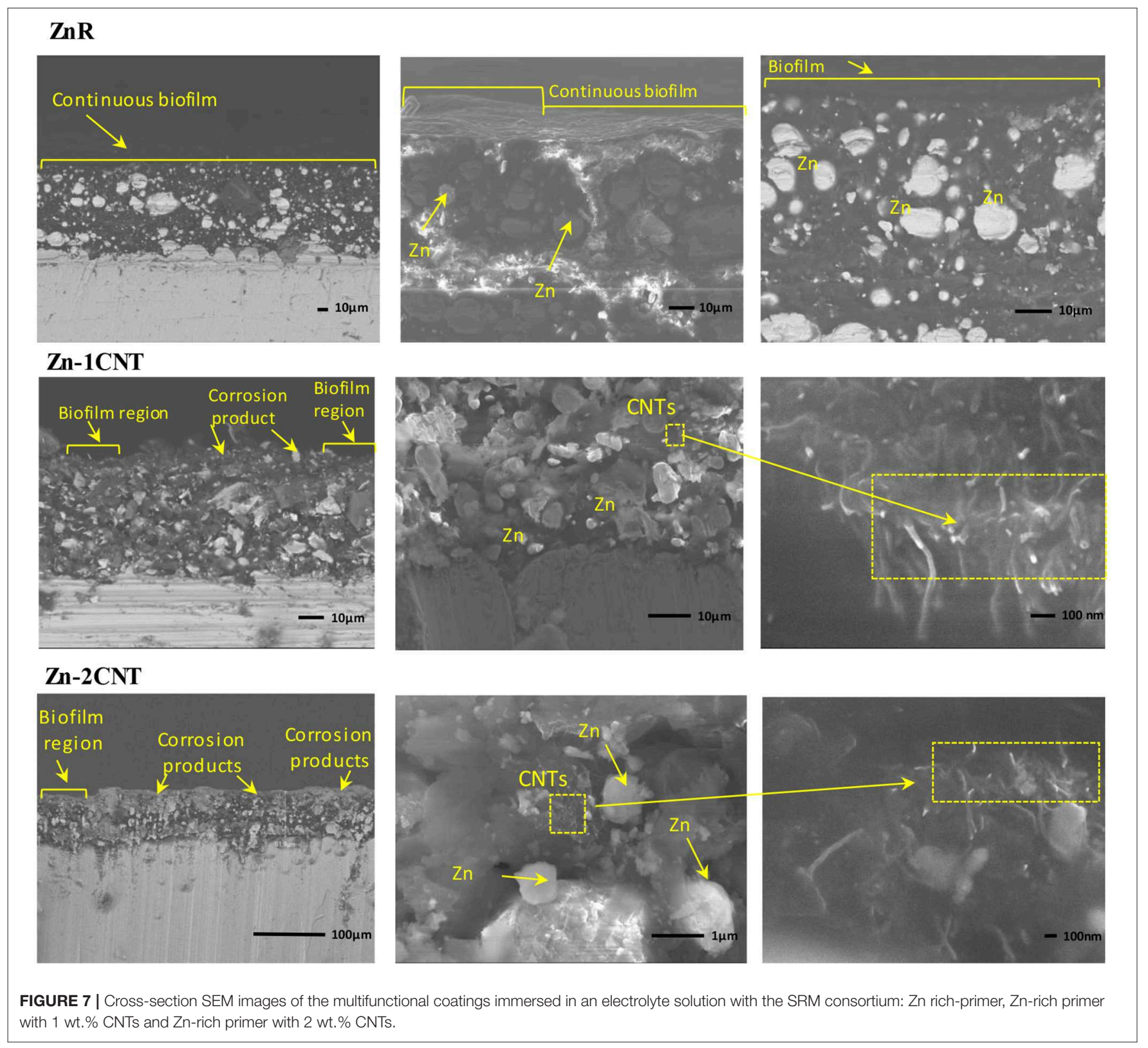

particles. Figure $\mathbf{8 b}$ illustrates the initial formation and lack of persistence of a layer of biofilm that shows some heterogeneous and residual over time. This finding is attributable to the inhibition of mature biofilm formation by the CNTs influence along with a poisoning mechanism by the cytotoxic effect of $\mathrm{Zn}$ soluble products (Babich and Stotzky, 1978; Bong et al., 2010) At this respect, CNTs are considered to have adverse effects on biofilm growth at some levels according to the investigations of Upadhyayula and Gadhamshetty (2010). When a double content of CNTs is present, a greater porosity can be appreciated as shown in Figure 8c, which is mainly attributed to the lack of continuous biofilm layer caused by an increased level of zinc corrosion products and the double content of carbon nanotubes that alter the development and formation of a mature biofilm.

Also, Figures 8a-c show the EDS analysis demonstrating that the $\mathrm{ZnR}$ coating has a higher content of organic compounds, such as carbon, sulfur, nitrogen, and phosphorous (C, S, N, and P) due to presence of a more mature biofilm, which supports the OCP data and impedance results. For the Zn-1CNT and Zn-2CNT coatings, a higher content of $\mathrm{Zn}$ and $\mathrm{O}$ is evident, and a lower level of organic compounds is found. Higher $\mathrm{Zn}$ levels indicate the formation of zinc corrosion products over the coating surface. In addition, the Zn-1CNT and Zn-2CNT coatings exhibit higher $\mathrm{Zn}$ and $\mathrm{O}$ content, thus further indicating that these coatings inhibit the formation of extracellular polymeric substances 

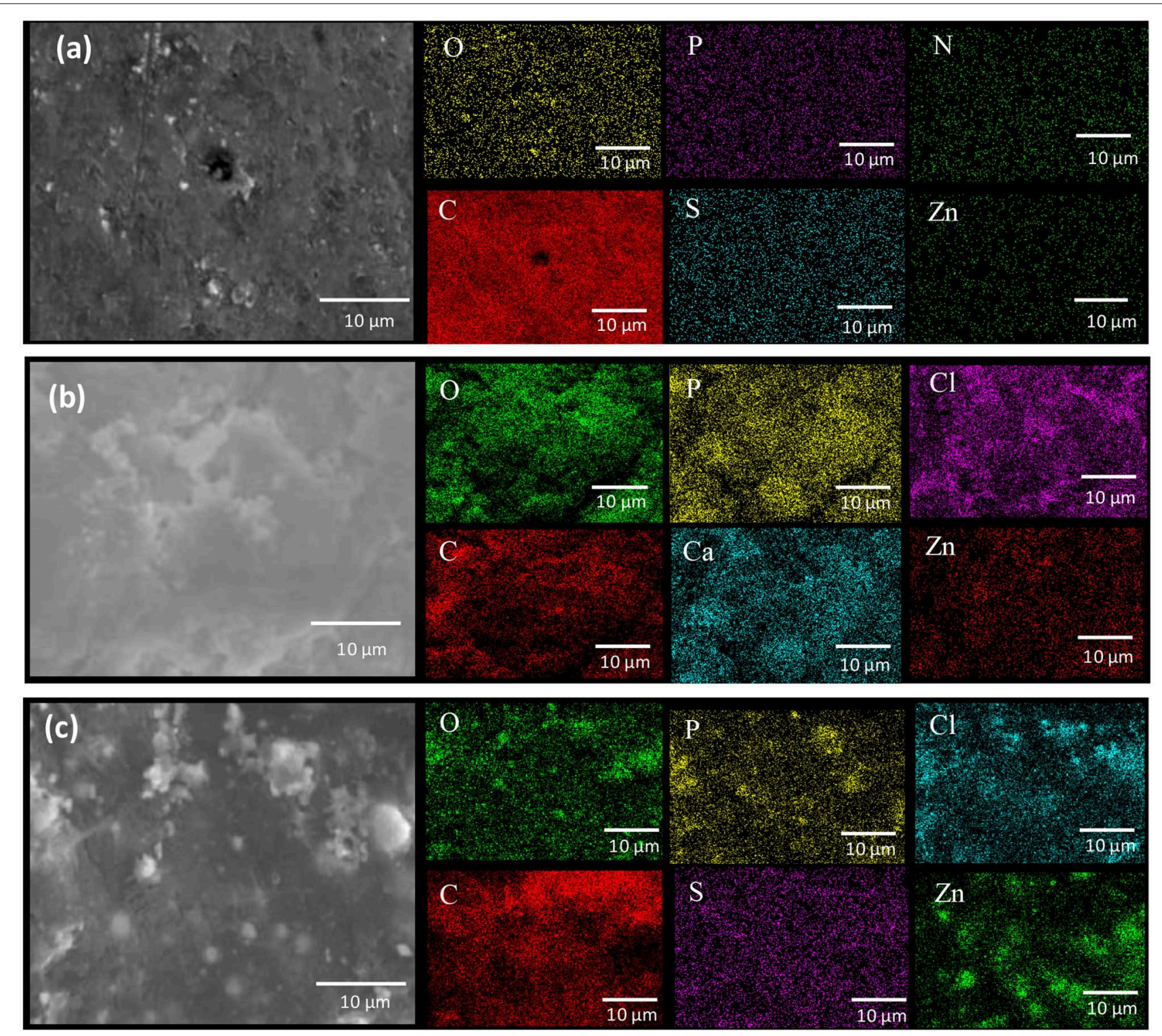

FIGURE 8 | Top views and EDS mapping analysis showing coatings samples after 29 days of exposure to the SRM consortium, (a) ZnR, (b) Zn-1CNT, and (c) Zn-2CNT. ZnR coating has a high content of organic compounds, such as carbon, sulfur, nitrogen and phosphorous (C, S, N, and P) due to biofilm formation.

(Babich and Stotzky, 1978; Bong et al., 2010; Billanger et al., 2015; Tong et al., 2015), and therefore disturb biofilm growth to a greater extent due to the toxicity of the high levels of CNTs (Upadhyayula and Gadhamshetty, 2010).

\section{CONCLUSIONS}

This investigation determined that for these multifunctional coatings, the anodic dissolution of zinc particles, the formation of an extra biolayer and the formation of zinc-rich corrosion products represent competing processes. The contribution of an extra layer formed on the coating surface can be attributed to the formation of EPSs and a biofilm by the SRM consortium.
The CNTs content enhances the anodic reactions of the zinc particles in the coatings, promoting cathodic protection and the formation of more corrosion products in the form of solid oxide/hydroxide compounds. However, doubling the CNT content results in more interaction with the zinc particles and impairment of biofilm growth. This represented a qualitative influence of CNTs concentration as evidenced by reduced levels of corrosion products. The $\mathrm{ZnR}$ coating exhibits galvanic protection, as evidenced by the formation of corrosion products and secretion of extracellular polymeric substances to produce a more homogeneous biolayer. The Zn-1CNT coating exhibits partial cytotoxicity toward microorganisms, thereby controlling biofilm initiation and growing processes. The $\mathrm{Zn}-2 \mathrm{CNT}$ ratio 
represents the saturated concentration of carbon nanotubes leading to less-optimal conditions for biofilm formation. Finally, we considered to be a CNTs content that could be used to balance biofilm formation and zinc anodic dissolution to influence microbiologically induced corrosion mechanisms.

\section{DATA AVAILABILITY STATEMENT}

All datasets generated for this study are included in the article/supplementary material.

\section{AUTHOR CONTRIBUTIONS}

HC: substantial contributions to the interpretation of data for the work, drafting the work content, final approval of the version to be published, and agreement to be accountable for all aspects of

\section{REFERENCES}

Abdolahi, A., Hamzah, E., Ibrahim, Z., and Hashim, S. (2014). Application of environmentally-friendly coatings toward inhibiting the microbially influenced corrosion (MIC) of steel: a review. Polym. Rev. 54, 702-745. doi: 10.1080/15583724.2014.946188

Abreu, C. M., Izquierdo, M., Perino, P., Novoa, X. R., and Pérez, C. (1999). A new approach to the determination of the cathodic protection period in Zinc-rich paints. Corrosion 55, 1173-1181. doi: 10.5006/1.3283955

Babich, H., and Stotzky, G. (1978). Toxicity of Zinc to fungi, bacteria, and coliphages: influence of chloride ions. Appl. Environ. Microbiol. 36, 906-914.

Billanger, X., Billard, P., Schneider, R., Balan, L., and Merlin, C. (2015). Stability and toxicity of $\mathrm{ZnO}$ quantumdots:Interplay between, nanoparticles and bacteria, J. Hazard Mater. 283, 110-116. doi: 10.1016/j.jhazmat.2014.09.017

Bong, C. W., Malfatti, F., Azam, F., Obayashi, Y., and Suszuki, S. (2010). The Effect of Zinc Exposure on the Bacteria Abundance and Proteolytic Activity in Seawater, Interdisciplinary Studies on Environmental Chemistry - Biological Responses to Contaminants (Tokyo: Terrapub), 57-63.

Bosch, R. W., Moons, F., Zheng, J. H., and Bogaerts, W. F. (2001). Application of electrochemical impedance spectroscopy for monitoring stress corrosion cracking. Corrosion 57, 532-539. doi: 10.5006/1.3290379

Castaneda, H., and Benetton, X. (2008). SRB-biofilm influence in active corrosion sites formed at the steel-electrolyte interface when exposed to artificial seawater conditions. Corr. Sci. 50, 1169-1183. doi: 10.1016/j.corsci.2007. 11.032

Ciubotariu, A., Benea, L., and Sand, W. (2015). "Effects of sulphate reducing bacteria on thermosetting polymer-Zn composite coatings," in "Mircea cel Batran" Naval Academy Scientific Bulletin, Vol. XVIII - 2015 (Constanta: "Mircea cel Batran" Naval Academy Press) (//PROQUEST SciTech Journals, PROQUEST Engineering Journals, Technology Journals, PROQUEST Military Collection PROQUEST Advanced Technologies and Aerospace).

Cubides, Y., and Castaneda, H. (2016). Corrosion protection mechanisms of carbon nanotube and zinc-rich epoxy primers on carbon steel in simulated concrete pore solutions in the presence of chloride ions. Corr. Sci. 109, 145-161. doi: 10.1016/j.corsci.2016.03.023

Deflorian, F., Rossi, S., Fedrizzi, L., and Bonora, P. L. (2005). EIS study of organic coating on zinc surface pretreated with environmentally friendly products, Progress in Organic Coatings 52, 271-279. doi: 10.1016/j.porgcoat.2004.04.005

Enning, D., and Garrelfs, J. (2014). Corrosion of iron by sulfate-reducing bacteria: new views of an old problem. Appl. Environ. Microbiol. 80, 1226-1236. doi: 10.1128/AEM.02848-13

Fedel, M., Rodríguez Gómez, F. J., Rossi, S., and Deflorian, F. (2019). Characterization of polyorganosilazane-derived hybrid coatings for the corrosion protection of mild steel in chloride solution. Coatings 9:680. doi: 10.3390/coatings9100680

Galicia, M., Valencia, V. G., and Aguirre-Ramírez, M., Castaneda, H. (2017). "Interfacial and Corrosion Characterization of Zinc Rich-Epoxy Primers with the work in ensuring that questions related to the accuracy or integrity of any part of the work are appropriately investigated and resolved. MG: substantial contributions to the design and set up conditions of the work and data acquisition (responsible of the laboratory where the work was made), participating in drafting the work and revising it critically for content, final approval of the version to be published, and agreement to be accountable for all aspects of the work.

\section{ACKNOWLEDGMENTS}

MG would like to thank Ivan Salcido for assistance with SEM images. Also, the authors would like to thank QBP Graciela García for a culture donation and Violeta Valencia for the electrochemical set up design.

Carbon Nanotubes Exposed to Marine Bacteria," in CORROSION 2017. New Orleans, LA: NACE International.

Guan, F., Zhai,X., Duan, J., and Zhang, M., Hou, B. (2016). Influence of sulfate-reducing bacteria on the corrosion behavior of high strength steel EQ70 under cathodic polarization. PLoS ONE 11:e0162315. doi: 10.1371/journal.pone.0162315

Jeon, H., Park, J., and Shon, M. (2013). Corrosion protection by epoxy coating containing multi-walled carbon nanotubes. J. Indus. Eng. Chem. 19, 849-853. doi: 10.1016/j.jiec.2012.10.030

Liu, D., Fe Lin, Q., Hui Li, C., and Li Xue, L. (2007). Improvement of MIC behavior of low alloy steel with Zn-rich epoxy coating. Key Eng. Mater. 348-349, 509-512. doi: 10.4028/www.scientific.net/KEM.348-349.509

Liu, T., and Cheng, Y. F. (2017). The influence of cathodic protection potential on the biofilm formation and corrosion behaviour of an X70 steel pipeline in sulfate reducing bacteria media. J. Alloys Compounds 729, 180-188. doi: 10.1016/j.jallcom.2017.09.181

Park, S. M., and Shon, M. Y. (2015). Effects of multi-walled carbon nanotubes on corrosion protection of zinc rich epoxy resin coating. J. Indus. Eng. Chem. 21, 1258-1264. doi: 10.1016/j.jiec.2014.05.042

Schwerdtfeger, W. J. (1958). Current and potential relation for the cathodic protection of steel in salt water. J. Res. Natl. Bureau Stand. 60, 153-159. doi: 10.6028/jres.060.019

Tong, T., Wilke, C. M., Wu, J., Binh, C. T., Kelly, J. J., and Gaillard, J. F. (2015). Combined toxicity of Nano- $\mathrm{ZnO}$ and $\mathrm{Nano}-\mathrm{TiO}_{2}$ : from single- to multinanomaterial systems. Environ. Sci. Technol. 49, 8113-8123. doi: 10.1021/acs.est.5b02148

Upadhyayula, V., and Gadhamshetty, V. (2010). Appreciating the role of carbon nanotube composites in preventing biofouling and promoting biofilms on material surfaces in environmental engineering: a review. Biotechnol. Adv. 28, 802-816. doi: 10.1016/j.biotechadv.2010.06.006

Wang, J., Li, Q. F., Fu, Y. D., and Li, C. H. (2012). MIC behdlftyavior of the low alloy steel with different Zn-epoxy coating in SRB solution. Key. Eng. Mater. 488-489, 262-265. doi: 10.4028/www.scientific.net/KEM.488-489.262

Weinell, C. E., and Rasmussen, S. N. (2007). Advancement in Zinc Rich Epoxy Primers for Corrosion Protection, in CORROSION 2007. Nashville, TN: NACE International.

Conflict of Interest: The authors declare that the research was conducted in the absence of any commercial or financial relationships that could be construed as a potential conflict of interest.

Copyright (C) 2019 Castaneda and Galicia. This is an open-access article distributed under the terms of the Creative Commons Attribution License (CC BY). The use, distribution or reproduction in other forums is permitted, provided the original author(s) and the copyright owner(s) are credited and that the original publication in this journal is cited, in accordance with accepted academic practice. No use, distribution or reproduction is permitted which does not comply with these terms. 